International Journal of Pure and Applied Mathematics

Volume $90 \quad$ No. $3 \quad 2014,281-285$

ISSN: 1311-8080 (printed version); ISSN: 1314-3395 (on-line version)

url: http://www.ijpam.eu

doi: http://dx.doi.org/10.12732/ijpam.v90i3.2

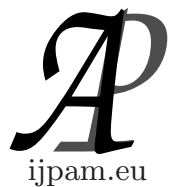

\title{
THE LAURENT SERIES FOR THE QUATERNIONIC CASE
}

\author{
M.F. Borges ${ }^{1 \S}$, J. Marão ${ }^{2}$ \\ ${ }^{1}$ UNESP - São Paulo State University \\ S.J. Rio Preto Campus \\ 15054-000, São José do Rio Preto, BRAZIL \\ ${ }^{2}$ Department of Mathematics \\ UFMA - Federal University of Maranhão \\ 65085-580, Maranhão, BRAZIL
}

\begin{abstract}
The present work has the scope to show the Laurent Series for quaternionic functions. It will be shown that the Laurent Series for the Quaternionic Case is analogous to the textbook case of Complex Analysis [1]-[2] already well established.
\end{abstract}

AMS Subject Classification: 30G99, 30E99

Key Words: Laurent series, hypercomplex functions, quaternionic functions

\section{Introduction}

The theory of hypercomplex functions mainly for quaternions and octonions, plays an important role in Mathematical Analysis, Geometry, as well as in Theoretical Physics as it reveals underlying aspects of symmetry in unifying Theories [1]-[2]. In recent days this theory presented analogous formulation to some well known results in the Complex Analysis [2], e.g trigonometric functions in hyp0ercomplex version, Taylor Series an of a Cauchy-Like Theorem for Quaternionic and Octonionic Functions [3], [8].

This work will be divided as follows:

Received: May 7, 2013

(C) 2014 Academic Publications, Ltd. url: www.acadpubl.eu

${ }^{\S}$ Correspondence author 
- The Cauchy Like Theorem for Quaternionic Functions;

- Laurent's Theorems for Complexa Variables;

- Laurent's Theorems for Quaternionic Functions.

The Cauchy-Like Theorem for Quaternionic Functions will be the key for determining the coefficients of the Laurent Series of the Qaternionic type and it is present below:

Theorem 1. Let $\Omega$ be a domain single sign in four-dimensional space and $f(q)$ be a regular function on $\Omega$ then

$$
\int_{\varphi} \frac{f(q)}{q-q_{0}} d q=\pi(i+j+2 k) f\left(q_{0}\right),
$$

where $\varphi$ is a simple closed hypersurface in $\Omega$ and $q_{0} \in \Omega$.

The Laurent series in the classical case is determined according to the following theorem [1] e [2]:

Theorem 2. If the annulus $\left(C_{1}, C_{2}\right)$ of center $z_{0}$ and understood in a void area $\left(C_{1}^{\prime}, C_{2}^{\prime}\right)$ in which the function $f(z)$ is analytic in the crown that is representable by a power series positive and negative $z-z_{0}$, call Laurent Series

$$
f(z)=\sum_{n=-\infty}^{\infty} a_{n}\left(z-z_{0}\right)^{n},
$$

whose coefficients, called Laurent coefficients are given by the Cauchy's formula:

$$
a_{n}=\frac{1}{2 \pi i} \oint_{C} \frac{f(z) d z}{\left(z-z_{0}\right)^{n+1}} .
$$

\section{The Laurent Series for Quaternionic Functions}

In order to fix ideas it is important to mention on the region that we will work the idea of Laurent Series for Quaternionic Case. The complex case predicts that the region will be crafted as a flat region. Thus, in the quaternionic case the region is conjectured as region of the four-dimensional space. Let us now assert the following theorem: 
Theorem 3. Be the region $\left(C_{1}, C_{2}\right)$ in four-dimensional space centered in $q_{0}$ and understood in a domain $\left(C_{1}^{\prime}, C_{2}^{\prime}\right)$ in which function $f(q)$ is an analytical, so this is representable in the region concerned by a power series of positive and negative $q-q_{0}$, call Laurent Series

$$
f(q)=\sum_{n=-\infty}^{\infty} a_{n}\left(q-q_{0}\right)^{n}
$$

and whose coefficients, called Laurent coefficients are given by the Cauchy formula for quaternionic functions [8]:

$$
a_{n}=\frac{1}{\pi(i+j+2 k)} \oint_{C} \frac{f(q) d q}{\left(q-q_{0}\right)^{n+1}}
$$

Demonstration: The proof is analogous to the complex case. Thus using Taylor's theorem we have that:

$$
f(q)=\frac{1}{\pi(i+j+2 k)} \oint_{C_{1}^{\prime}} \frac{f(t) d t}{t-q}-\frac{1}{\pi(i+j+2 k)} \oint_{C_{2}^{\prime}} \frac{f(t) d t}{t-q}
$$

assuming that the surface $C_{2}^{\prime}$ is traversed in the positive direction, $C_{1}^{\prime}$ in the negative sense. Thus, as in the Taylor series, we have to $C_{1}^{\prime}$

$$
\frac{1}{\pi(i+j+2 k)} \oint_{C_{1}^{\prime}} \frac{f(t) d t}{t-q}=\sum_{n=0}^{\infty} a_{n}\left(q-q_{0}\right)^{n}
$$

whose coefficients are given by:

$$
a_{n}=\frac{f^{n}\left(q_{0}\right)}{n !}=\frac{1}{\pi(i+j+2 k)} \oint_{C_{1}^{\prime}} \frac{f(t) d t}{\left(t-q_{0}\right)^{n+1}} .
$$

Now follows a case in which the integration is performed by $C_{2}^{\prime}$. Therefore, we write:

$$
\frac{1}{t-q}=-\frac{1}{q-q_{0}}\left(1-\frac{t-q_{0}}{q-q_{0}}\right)^{-1}
$$

and considering that now:

$$
\left|\frac{t-q_{0}}{q-q_{0}}\right|<1
$$

follows immediately that

$$
\left(1-\frac{t-q_{0}}{q-q_{0}}\right)^{-1}=\sum_{m=0}^{\infty}\left(\frac{t-q_{0}}{q-q_{0}}\right)^{m} .
$$


Similarly to the previous case, we can do:

$$
-\frac{1}{\pi(i+j+2 k)} \oint_{C_{2}^{\prime}} \frac{f(t)}{t-q}=\sum_{n=-1}^{-\infty}\left(q-q_{0}\right)^{n} \frac{1}{\pi(i+j+2 k)} \oint_{C_{2}^{\prime}} \frac{f(t)}{\left(t-q_{0}\right)^{n+1}}
$$

which gives us:

$$
f(z)=\sum_{n=0}^{\infty} a_{n}\left(q-q_{0}\right)^{n}+\sum_{n=-1}^{-\infty} a_{n}\left(q-q_{0}\right)^{n}
$$

with $n>0$ is a valid expression:

$$
a_{n}=\frac{1}{\pi(i+j+2 k)} \oint \frac{f(t) d t}{\left(t-q_{0}\right)^{n+1}}
$$

\section{Conclusion}

In this brief report the Laurent Series for Quaternionic Functions have been determined it will be important in quaternionic functions that will be addressed in future works, for instance:

- Deepening Integration Theory of Functions Hypercomplex, quaternionic functions in particular;

- Formulation of the Theory of Functions Waste for Quaternions;

- Implementation and resolution of Quadruples Improper integrals.

The topics listed above will also be key for solving physical problems which comes from a formulation in four dimensions, and support in problems relating to the consolidation of the Theory of Hypercomplex Functions, a goal of the research so far.

\section{References}

[1] J.H. Conway, D.A. Smith, On Quaternions and Octonions: Their Geometry Arithmetica and Symetry, A.K. Peters, Batiek, MA, 20, No. 1 (2003), 159pp; 
[2] Kunihiko Kodaira, Complex Analysis, Cambridge Studies in Advanced Mathematics, Cambridge University Press Cambridge (2007), 406pp.

[3] J.M. Machado, M.F. Borges, Hypercomplex functions and conformal mappings, Internacional Journal of Applied Math., 1 (2002), 27-38.

[4] J.B. Maricato, J.M. Machado, M.F. Borges, Quasiconformal transformations and hypercomplex functions, Internacional Journal of Applied Math., 20 (2007), 691-702.

[5] M.F Borges, L.F. Benzatti, Quasiconformal mappings in octonionic algebra: A graphical an analytical compareson, Far East Journal of Mathematical Sciences, 33 (2009), 355-361.

[6] M.F. Borges, J.A. Marão, J.M. Machado, Geometrical octonions I: On trigonometric and logarithmic functions of octonionic type, Internacional Journal of Applied Math., 21 (2008), 461-471.

[7] M.F. Borges, J.A. Marão, J.M. Machado, Geometrical octonions II: Hyper regularity and hyper periodicity of the exponential function, Internacional Journal of Pure and Applied Math., 48 (2008), 495-500.

[8] M.F. Borges, J.A. Marão, R.C. Barreiro, A Cauchy-Like theorem for hypercomplex functions, Journal of Geometry and Topology, 3 (2009), 263-271. 
